\title{
Occurrence of Diarrheal diseases among under-five children and associated socio-demographic and household environmental factors: An Investigation Based on National Family Health Survey-4 in Rural India
}

Jay Saha ( $\sim$ jsaha519@gmail.com )

University of Gour Banga (UGB)

Sabbir Mondal

University of Gour Banga (UGB)

Pradip Chouhan

University of Gour Banga (UGB)

Research Article

Keywords: Diarrheal disease, Under-five children, Environmental Factors, NFHS, Rural India

Posted Date: December 21st, 2021

DOI: https://doi.org/10.21203/rs.3.rs-1179622/v1

License: (c) (1) This work is licensed under a Creative Commons Attribution 4.0 International License.

Read Full License 


\section{Abstract}

Background: Diarrheal disease is a major population health problem that is the leading reason for mortality and morbidity among children aged 0-59 months in rural India. Therefore, the rationale of this study was to identify the socio-demographic, environmental predictors associated with diarrhea among under-five children in rural India. Methods: A total of 188,521 living children (0-59 months) were utilized from the National Family Health Survey-4, 2015-2016. Bivariate and binary logistic regression analysis was carried out from the available NFHS-4 data for selected socio-demographic and environmental predictors to identify the relationship of occurrence of diarrhea using STATA 13.1. Results: In rural India, children aged $12-23$ months [AOR: $0.897,95 \% \mathrm{Cl}(0.876,0.983)$ ], $24-35$ months [AOR: $0.579,95 \% \mathrm{Cl}$ $(0.543,0.617)$ ], $36-47$ months [AOR: $0.394,95 \% \mathrm{Cl}(0.367,0.424)], 48-59$ months [AOR: $0.313,95 \% \mathrm{Cl}$ $(0.289,0.339)]$ were significantly less likely to suffer diarrheal disease. Female children [AOR: $0.897,95 \%$ $\mathrm{Cl}(0.859,0.937)]$, children belonged to Scheduled Tribe [AOR: $0.811,95 \% \mathrm{Cl}(0.755,0.872)]$, Other Backward Classes [AOR: $0.902,95 \% \mathrm{Cl}(0.851,0.956)$ ] were less likelihood to experience diarrhea significantly. Diarrhea disease was also significantly more likely to occur among Muslim children [AOR: $1.217,95 \% \mathrm{Cl}(1.128,1.313)]$, other religion [AOR: $1.163,95 \% \mathrm{Cl}(1.062,1.272)]$ children in central region [AOR: 1.510, 95\% Cl (1.410, 1.617)], east region [AOR: 1.077, 95\% Cl $(1.002,1.157)$ ], and west region [AOR: $1.201,95 \% \mathrm{Cl}(1.095,1.317)]$, children with low birth weight [AOR: 1.135, 95\% Cl $(1.074,1.149)$ ], undernourished [AOR: 1.097, 95\% Cl (1.038, 1.197)], improper stool disposal [AOR: $1.061,95 \% \mathrm{Cl}(1.002$, 1.124)], and rudimentary roof materials [AOR: $1.113,95 \% \mathrm{Cl}(1.048,1.182)]$. Conclusions: In the rural part of India, diarrhea has occurred frequently now. The different socio-demographic and environmental factors are influencing this disease. For reducing the vulnerability of diarrhea the socio-demographic and environmental factors should be improved or monitoring by effective community education. The government and different NGOs should focus on improved drinking water sources, sanitation facility which may reduce the vulnerability of the disease.

\section{Introduction}

Worldwide, Diarrheal disease is a major population health hazard which is leading cause of mortality and morbidity among children aged 0-59 months as well as in developing countries According to WHO, Diarrhea is defined as the passage of three or more loose or liquid stools per day (or more frequent passage than is normal for the individual [1]. Diarrhea is usually a symptom of an infection in the intestinal tract, which can be caused by a variety of bacterial, viral, and parasitic organisms $[1,2]$. The main causes of diarrhea deaths were severe dehydration and fluid loss for most people and undernourished or have impaired immunity children are most at risk of life-threatening diarrhea [1]. Diarrhea should be treated with an oral rehydration solution (ORS), a solution of clean water, sugar, and salt [3].

Despite the availability of a simple treatment solution, diarrhea remains a leading killer of young children, 3 out of 1000 children under-five years died due to diarrhea in 2016, accounting for approximately 8 per cent of all deaths among children under age 5 worldwide in 2017 [4], the second leading cause of death 
in children under five years old i.e. Globally, there are nearly 1.7 billion cases of childhood diarrhoeal disease every year and killed around 525,000 under-five children [1]. Most deaths from diarrhea develop among children less than 2 years of age living in South Asia and sub-Saharan Africa [5]. From 2000-2017, the total annual number of deaths from diarrhea among children under 5 decreased by 60 per cent [6]. In India was similar, with an under-five mortality rate due to diarrhea of 4 per 1000 live births in 2016, representing $9 \%$ of total deaths among under-five children [6]. In India, according to National Family Health Survey (NFHS)-4 report, $9.19 \%$ of children (8.24\% in urban and $9.57 \%$ in rural) under age five years had diarrhea in the two weeks before the survey [7]. Children in rural India are treated wrongly for diarrhea and pneumonia, which are the two leading killers of young ones worldwide [8]. According to the NFHS-4 report, the occurrence of diarrhoeal disease rises from $11 \%$ among children under age 6 months to $16 \%$ among those aged 6-11 months in 2015-16 [7].

Pneumonia and diarrhea together account for $29 \%$ of all child deaths globally [9], $50 \%$ of all child deaths in India [10]. The Integrated Global Action Plan for the Prevention and Control of Pneumonia and Diarrhoea (GAPPD) was launched by WHO/UNICEF, to reduce these two preventable diseases i.e. diarrhea and pneumonia [9]. In WHO/ UNICEF report 'Diarrhoea: Why children are still dying and what can be done, lays out a 'seven-point plan' that includes a treatment package to reduce childhood diarrhoea deaths and a prevention strategy to ensure long-term results $[11,12]$. To reduce diarrheal disease deaths in the country, the union health ministry in association with a few international health organizations has launched the 'Intensified Diarrhea Control Fortnight-2015 program' in India [13]. Accredited Social Health Activists (ASHA) workers will carry out various activities in the remote and distant parts of the states for distribution of ORS packets to every family with children under age five along with group counseling [14]. The Integrated Child Development Services (ICDS) scheme is the largest program launched by the Government of India on 2nd October 1975 for the encouragement of maternal and child health and nutrition not only in India to reduce childhood malnutrition and associated morbidities among Under-five children [15].

Several child socio-demographics, nutritional, health, and environmental predictors are associated directly and indirectly with the occurrence of diarrheal disease among under-five children. These sociodemographics, nutritional, health factors include child's sex, child's age, maternal education, maternal age, household economic [16, 17, 26-28, 18-25], breastfeeding status, and nutritional status [17, 20, 23, 26]. Environmental predictors include drinking water quality, sanitation condition, stool disposal site, and household dwelling characteristics [16, 23, 24, 26, 27, 29-31].

The 2015-16 National Family and Health Survey report showed that under-five children in rural India had, relatively, more diarrhea occurrences than urban ones. Even though a high level of diarrhea disease in rural India, there are few studies conducted at the District level to assess diarrhoeal disease and its associated factors. But, there is no study at the country level, which pays attention specifically to the rural part of the country, using a nationally representative survey to show associated factors of diarrhea occurrence among under-five children. So, evidence-based information is needed for a child's health development strategy by preventing and dropping the severity of diarrhoeal in under-five children in rural 
India. Thus, this study was intended to fill this gap by achieving its aim which was to identify the sociodemographic, and environmental associated factors of the occurrence of diarrhea among children under-five years in rural India.

\section{Data And Methods}

\subsection{Study design and period}

A cross-sectional study was conducted using the latest round of NFHS. Data were collected between January 20, 2015, and December 4, 2016.

\subsection{Data source and study sample}

For this study, data was used from the fourth round of the National Family Health Survey, 2015-16, consisting of nationally representative sample surveys. The purpose of this survey was to gather essential information on family planning, fertility, maternal and child health, under-5 nutrition, anemia, infant, child, and maternal mortality indicators, other adult health issues HIV/AIDS-related knowledge, attitudes, and behavior, domestic violence [7].

A total of 188,521 living children (0-59 months) were utilized from the National Family Health Survey NFHS-4 in rural India from different socio-demographic and environmental characteristics of under-five children. The data used in this study were retrieved from the public domain after describing the objective of the study.

\subsection{Sampling design and technique}

The NFHS-4 samples were collected using a stratified two-stage sampling design. In the first stage of sample selection, 28,586 Primary Sampling Units (PSUs) were selected, 130 PSUs were selected from the slums which are listed by Municipal Corporation Offices (MCOs), and the rest were selected from the census sampling frame (8,397 in urban areas and 20,059 in rural areas) [7]. The rural sample was selected through a two-stage sample design with villages as the PSUs (selected with probability proportional to size [PPS]) for rural sample, and followed by a random selection of 22 households in each PSU in rural areas at the second stage; households were selected after conducting a complete mapping and household listing operation in the selected first-stage units [7].

\subsection{Outcome variable}

'Had diarrhea recently' is the outcome variable of this study. It is defined as having three or more loose or watery stools in 24 hours, as reported by the mother/caretaker of the child in the past 2 weeks preceding the data collection. This variable was coded as 'no' diarrhea (coded as ' 0 ') and 'yes' diarrhea (assigned as code ' 1 ') for the smooth running of the bivariate and multivariate logistic regression model

\subsection{Predictor variables}




\subsubsection{Socio-demographic predictors}

For the analysis of the study, a selection of socio-demographic and environmental predictor variables were included in this study which was identified from the pre-existing literature study and associated with the diarrhoeal disease among under-five children. Children's socio-demographic predictor variables include social groups [Scheduled Caste (SC), Scheduled Tribe (ST), Other Backward Classes (OBC), and Other], religion (Hindu, Muslim, and Other), current age of the mother in years (classified into 3 groups: 15-24, 25-34, and 35-49 years), education of mother/caregivers (illiterate \& primary and secondary \& above), wealth index (poor, middle, and rich), Body Mass Index (BMI) of mother (underweight $[<18.5$ $\left.\mathrm{kg} / \mathrm{m}^{2}\right]$, normal weight [18.5-24.9 kg/m²], and overweight \& obesity [>25 kg/m²]), region (north, central, east, north-east, west, and south), child's sex (male and female), current age of child in months (0-11, 12-23, 24-35, 36-47, and 48-59 months), birth order number (1, 2-3, and 4 and more) and birth weight (normal [ $\geq 2500 \mathrm{gm}$ ] and low birth weight [ $<2500 \mathrm{gm}]$, nutritional status (based on WHO Multicentre Growth Reference Study weight-for-age of the under-five children was computed, then nutritional status is classifies as normal [above minus two standard deviations (-2SD)] and under nourished [below minus two standard deviations (-2SD) and minus three standard deviations (-3SD) from the median of the reference population]), and currently breastfeeding (no and yes).

\subsubsection{Household environmental predictors}

Child's environmental predictor variables include sources of drinking water (unimproved and improved [Include piped water, public taps, standpipes, tube wells, boreholes, protected dug wells and springs, rainwater, and community reverse osmosis (RO) plants [7]]), toilet facilities (unimproved and improved [Include any non-shared toilet of the following types: flush/pour flush toilets to piped sewer systems, septic tanks, and pit latrines; ventilated improved pit (VIP)/biogas latrines; pit latrines with slabs; and twin pit/composting toilets [7]]), toilet facility shared with other households (not shared and shared), disposal of youngest child's stools when not using the toilet (proper disposal [properly disposed of, if a child used a toilet or latrine, or put/rinsed into a toilet, or it was buried [7]] and improper disposal), main roof material (concrete and rudimentary roof), and main floor material (clean and unclean).

\subsection{Statistical analyses}

All the analyses were completed using the Statistical Package and Data Science software STATA version 13.1 (StataCorp LP, College Station, TX, USA). In the preliminary section of the analysis, inferential data analysis and general descriptive analysis have been carried out to explain the weighted percentage of sample children, and also the number of living children (total sample, $n$ ) with their socio-demographic and environmental characteristics. The sample survey data were weighted to represent the under-five children's structure of rural India, using the weighting factors provided by the NFHS. To explain the occurrence of diarrhea differentials, bivariate association, and Pearson's chi-square test analyses were used. A multivariate binary logistic regression model was used to identify the socio-demographic and environmental predictors associated with the occurrence of diarrhea among children aged 0-59 months. The power of the associations between predictors and the outcome variable in the analysis depended on 
Crude Odds Ratios (COR) and Adjusted Odds Ratios (AOR) with 95\% confidence intervals, and the level of significance at $(P$-value $<0.05)$.

\section{Results}

\subsection{Socio-demographic characteristics of the living under- five children}

In this study, a total of 188,521 living children were incorporated aged 0-59 months in rural India. The majority, of children (81.49\%) religion was Hindu and nearly one-fourth of the children $(23.77 \%)$ belonged to Scheduled Caste (SC). The majority of mothers (54.73\%) were in the age group of 25-34 years, half of the mother/caregivers (50.27\%) had no education to the primary level of education, nearly one-fourth $(28.22 \%)$ of them were underweight. Of the total $29.63 \%$ and $28.88 \%$ of children were from the east and central region respectively. Near about half of the mothers (47.49\%) had 2-3 numbers of birth orders and more than half of the children's families $(59.89 \%)$ had poor wealth conditions i.e. economically poor. Among living under-five children, $51.88 \%$ were male children, $38.25 \%$ of children were undernourished, and $17.88 \%$ had low birth weight, and $68.48 \%$ of children had a history of breastfeeding currently (Table 1). 
Table 1

Socio-demographic characteristics of the living children aged 0-59 months in Rural India, NFHS-4, 2015-16

\begin{tabular}{|c|c|c|}
\hline Variables & Frequency (n) & Percentage (\%) \\
\hline \multicolumn{3}{|l|}{ Social groups } \\
\hline SC & 36,619 & 23.77 \\
\hline ST & 42,684 & 13.37 \\
\hline OBC & 71,685 & 45.91 \\
\hline Other & 28,941 & 16.95 \\
\hline \multicolumn{3}{|l|}{ Religion } \\
\hline Hindu & 139,770 & 81.49 \\
\hline Muslim & 25,532 & 13.88 \\
\hline Other & 23,219 & 4.63 \\
\hline \multicolumn{3}{|c|}{ Current age of mother in years } \\
\hline $15-24$ & 63,908 & 36.75 \\
\hline $25-34$ & 105,668 & 54.73 \\
\hline $35-49$ & 18,945 & 8.52 \\
\hline \multicolumn{3}{|c|}{ Education of mother/caregivers } \\
\hline Illiterate \& primary & 95,541 & 50.27 \\
\hline Secondary \& above & 92,980 & 49.73 \\
\hline \multicolumn{3}{|l|}{ Wealth index } \\
\hline Poor & 113,792 & 59.89 \\
\hline Middle & 38,361 & 20.68 \\
\hline Rich & 36,368 & 19.43 \\
\hline \multicolumn{3}{|l|}{ Maternal BMI } \\
\hline Underweight & 48,629 & 28.22 \\
\hline Normal & 118,211 & 61.20 \\
\hline Overweight \& obesity & 19,634 & 10.59 \\
\hline \multicolumn{3}{|l|}{ Region } \\
\hline North & 34,556 & 12.19 \\
\hline
\end{tabular}




\begin{tabular}{|c|c|c|}
\hline Variables & Frequency $(n)$ & Percentage (\%) \\
\hline Central & 54,593 & 28.88 \\
\hline East & 43,904 & 29.63 \\
\hline North-east & 28,213 & 4.21 \\
\hline West & 11,663 & 10.00 \\
\hline South & 15,592 & 14.84 \\
\hline \multicolumn{3}{|l|}{ Sex of child } \\
\hline Male & 97,625 & 51.88 \\
\hline Female & 90,896 & 48.12 \\
\hline \multicolumn{3}{|c|}{ Current age of child in months } \\
\hline $0-11$ & 37,207 & 19.70 \\
\hline $12-23$ & 37,489 & 20.02 \\
\hline $24-35$ & 37,326 & 19.77 \\
\hline $36-47$ & 39,028 & 20.67 \\
\hline $48-59$ & 37,471 & 19.84 \\
\hline \multicolumn{3}{|c|}{ Birth order number } \\
\hline 1 & 67,038 & 36.46 \\
\hline $2-3$ & 88,217 & 47.49 \\
\hline $3+$ & 33,266 & 16.06 \\
\hline \multicolumn{3}{|l|}{ Birth weight } \\
\hline Normal & 112,591 & 82.12 \\
\hline Low birth weight & 23,423 & 17.88 \\
\hline \multicolumn{3}{|l|}{ Nutritional status } \\
\hline Normal & 109,124 & 61.75 \\
\hline Undernourished & 62,395 & 38.25 \\
\hline \multicolumn{3}{|c|}{ Currently breastfeeding } \\
\hline No & 59,215 & 31.52 \\
\hline Yes & 129,306 & 68.48 \\
\hline
\end{tabular}




\subsection{Household environmental characteristics of the living under-five children}

Concerning the environmental predictors, near about one-tenth of children (10.24\%) used drinking water from unimproved sources, about $37.19 \%$ of children had improved toilet facilities, $17.16 \%$ of children had shared toilet facilities with other households, and about one-third of children (75.89\%) children disposed of their stools improperly. Furthermore, of the total children, $56.55 \%$ had a dwelling with an unclean floor and $17 \%$ of children had a rudimentary roof in rural India. (Table 2 )

Table 2

Household environmental characteristics of the living children aged 0-59 months in Rural India, NFHS-4, 2015-16

\begin{tabular}{|lll|}
\hline Environmental characteristics & Frequency (n) & Percentage (\%) \\
\hline Sources of drinking water & 23,844 & 10.24 \\
\hline Unimproved & 154,383 & 89.76 \\
\hline Improved & & \\
\hline Toilet facilities & & 62.81 \\
\hline Unimproved & 96,059 & 37.19 \\
\hline Improved & 71,075 & \\
\hline Toilet facility shared with other h. h. & & 82.84 \\
\hline Not shared & 69,191 & 17.16 \\
\hline Shared & 13,290 & \\
\hline Disposal of youngest child's stools & & 24.11 \\
\hline Proper disposal & 49,544 & 75.89 \\
\hline Improper disposal & 136,140 & \\
\hline Main roof material & & 83.00 \\
\hline Concrete roof & 142,676 \\
\hline Rudimentary roof & 28,601 & 17.00 \\
\hline Main floor material & & \\
\hline Clean & 100,212 & \\
\hline Unclean & & \\
\hline
\end{tabular}




\subsection{Occurrence of diarrhea according to different socio- demographic and environmental predictors}

The total occurrence of diarrhea among under-five children was $9.57 \%$ in rural India. The result in Table 3 showed that the development of diarrhea was higher among the children whose mothers/caregivers had no education to the primary level of education; children belonged to Scheduled Caste (9.89\%), and children belonged to Muslim religion (10.32\%). The prevalence of diarrhea was higher among those children's mothers who were aged 15-24 years (10.9\%) and with an underweight body mass index (10.19\%). The maximum occurrence of diarrhea was also found among the children from the central region $(14.3 \%)$, those children from the poor wealth index (9.97\%). The occurrence of diarrhea was the highest among children aged 0-11 months (14.41\%) and 12-23 months (13.89\%), male children (9.88\%), currently breastfeeding $(10.55 \%)$, with birth order more than three $(10.6 \%), 10.76 \%$ and $10.37 \%$ of children had more occurrence of diarrhea with low birth weight and undernourished nutritional status respectively. 
Table 3

Occurrence of diarrheal diseases by socio-demographic characteristics of respondents in Rural India, NFHS-4, 2015-16.

\begin{tabular}{|c|c|c|c|c|}
\hline \multirow[t]{2}{*}{ Socio-demographic characteristics } & \multicolumn{2}{|c|}{ Had Diarrhoea recently } & \multirow[t]{2}{*}{ Pearson's $\chi 2$ value } & \multirow[t]{2}{*}{ p-value } \\
\hline & No $(\%)$ & Yes (\%) & & \\
\hline Social groups & & & 232.06 & $<0.001$ \\
\hline SC & 90.11 & 9.89 & & \\
\hline ST & 91.67 & 8.33 & & \\
\hline $\mathrm{OBC}$ & 90.12 & 9.88 & & \\
\hline Other & 90.26 & 9.74 & & \\
\hline Religion & & & 223.84 & $<0.001$ \\
\hline Hindu & 90.43 & 9.57 & & \\
\hline Muslim & 89.68 & 10.32 & & \\
\hline Other & 92.84 & 7.16 & & \\
\hline Current age of mother in years & & & 256.40 & $<0.001$ \\
\hline $15-24$ & 89.10 & 10.90 & & \\
\hline $25-34$ & 91.19 & 8.81 & & \\
\hline $35-49$ & 91.35 & 8.65 & & \\
\hline Education of mother/caregivers & & & 0.1122 & 0.738 \\
\hline Illiterate \& primary & 90.27 & 9.73 & & \\
\hline Secondary \& above & 90.6 & 9.40 & & \\
\hline Wealth index & & & 7.90 & 0.01 \\
\hline Poor & 90.03 & 9.97 & & \\
\hline Middle & 90.56 & 9.44 & & \\
\hline Rich & 91.55 & 8.45 & & \\
\hline Maternal BMI & & & 28.39 & $<0.001$ \\
\hline Underweight & 89.81 & 10.19 & & \\
\hline Normal & 90.55 & 9.45 & & \\
\hline Overweight \& obesity & 91.36 & 8.64 & & \\
\hline Region & & & $1.30 \mathrm{E}+03$ & $<0.001$ \\
\hline
\end{tabular}




\begin{tabular}{|c|c|c|c|c|}
\hline \multirow[t]{2}{*}{ Socio-demographic characteristics } & \multicolumn{2}{|c|}{ Had Diarrhoea recently } & \multirow[t]{2}{*}{ Pearson's $\chi 2$ value } & \multirow[t]{2}{*}{ p-value } \\
\hline & No $(\%)$ & Yes (\%) & & \\
\hline North & 92.33 & 7.67 & & \\
\hline Central & 86.82 & 13.18 & & \\
\hline East & 90.85 & 9.15 & & \\
\hline North-east & 95.96 & 4.04 & & \\
\hline West & 90.47 & 9.53 & & \\
\hline South & 93.47 & 6.53 & & \\
\hline Sex of child & & & 31.70 & $<0.001$ \\
\hline Male & 90.12 & 9.88 & & \\
\hline Female & 90.77 & 9.23 & & \\
\hline Current age of child in months & & & $3.00 \mathrm{E}+03$ & $<0.001$ \\
\hline $0-11$ & 85.59 & 14.41 & & \\
\hline $12-23$ & 86.11 & 13.89 & & \\
\hline $24-35$ & 91.08 & 8.92 & & \\
\hline $36-47$ & 94.01 & 5.99 & & \\
\hline $48-59$ & 95.25 & 4.75 & & \\
\hline Birth order number & & & 12.85 & 0.002 \\
\hline 1 & 90.80 & 9.20 & & \\
\hline $2-3$ & 90.50 & 9.50 & & \\
\hline $3+$ & 89.40 & 10.6 & & \\
\hline Birth weight & & & 78.32 & $<0.001$ \\
\hline Normal & 90.96 & 9.04 & & \\
\hline Low birth weight & 89.24 & 10.76 & & \\
\hline Nutritional status & & & 42.29 & $<0.001$ \\
\hline Normal & 90.74 & 9.26 & & \\
\hline Undernourished & 89.63 & 10.37 & & \\
\hline Currently breastfeeding & & & 355.03 & $<0.001$ \\
\hline No & 92.58 & 7.42 & & \\
\hline
\end{tabular}




\begin{tabular}{|c|c|c|c|c|}
\hline \multirow[t]{2}{*}{ Socio-demographic characteristics } & \multicolumn{2}{|c|}{ Had Diarrhoea recently } & \multirow[t]{2}{*}{ Pearson's $\chi 2$ value } & \multirow[t]{2}{*}{ p-value } \\
\hline & No (\%) & Yes (\%) & & \\
\hline Yes & 89.45 & 10.55 & & \\
\hline
\end{tabular}

Note: NFHS-4, Rural India, 2015-16. Percentages are computed by applying sample weight

\subsection{Occurrence of diarrhea according to different household environmental characteristics}

In rural India, diarrheal diseases were also found remarkably higher among those children whose households have unimproved toilet facilities (10.06\%) compared to those households which have improved toilet facilities (8.84\%), and diarrhea also significantly higher among those children had shared $(10.1 \%)$ their toilet facility with other households than those were not shared (8.36\%). Astonishingly, the occurrence of diarrhea was higher among children whose households had used improved sources $(9.69 \%)$ of drinking water than those children who had used unimproved sources $(7.74 \%)$ of drinking water. The occurrence of diarrhea was significantly higher among the children dwelling with the unclean floor (10.29\%) and children with rudimentary roof materials (10.4\%) (Table 4). 
Table 4

Occurrence of diarrheal diseases by household environmental characteristics of respondents in Rural India, NFHS-4, 2015-16.

\begin{tabular}{|c|c|c|c|c|}
\hline \multirow[t]{2}{*}{ Environmental characteristics } & \multicolumn{2}{|c|}{ Had Diarrhoea recently } & \multirow[t]{2}{*}{ Pearson's $\chi 2$ value } & \multirow[t]{2}{*}{ p-value } \\
\hline & No (\%) & Yes (\%) & & \\
\hline Sources of drinking water & & & 82.08 & $<0.001$ \\
\hline Unimproved & 92.26 & 7.74 & & \\
\hline Improved & 90.31 & 9.69 & & \\
\hline Toilet facilities & & & 54.04 & $<0.001$ \\
\hline Unimproved & 89.94 & 10.06 & & \\
\hline Improved & 91.16 & 8.84 & & \\
\hline Toilet facility shared with other $h . h$. & & & 63.24 & $<0.001$ \\
\hline Not shared & 91.64 & 8.36 & & \\
\hline Shared & 89.90 & 10.10 & & \\
\hline Disposal of youngest child's stools & & & 91.32 & $<0.001$ \\
\hline Proper & 92.30 & 7.70 & & \\
\hline Improper & 89.85 & 10.15 & & \\
\hline Main roof material & & & 35.74 & $<0.001$ \\
\hline Concrete roof & 90.79 & 9.21 & & \\
\hline Rudimentary roof & 89.43 & 10.57 & & \\
\hline Main floor material & & & 60.54 & $<0.001$ \\
\hline Clean & 91.54 & 8.46 & & \\
\hline Unclean & 89.71 & 10.29 & & \\
\hline Rural India & 90.43 & 9.57 & & \\
\hline
\end{tabular}

\section{5 socio-demographic predictors associated with diarrhea among under-five children}

In Table 5, the Multivariate logistic regression method was used to evaluate the relative effect of the predictor variable on the outcome variable. The incidence of childhood diarrhea had significantly 0.811 and 0.902 times less likely to occur among the children belonged to Scheduled Tribe [AOR: $0.811,95 \% \mathrm{Cl}$ $(0.755,0.872)]$, Other Backward Classes [AOR: $0.902,95 \% \mathrm{Cl}(0.851,0.956)]$ respectively compared to 
Scheduled Caste children. The diarrhoeal disease was also significantly 1.217 and 1.163 times more likely to happen among Muslim children [AOR: $1.217,95 \% \mathrm{Cl}(1.128,1.313)]$, and other religious groups [AOR: $1.163,95 \% \mathrm{Cl}(1.062,1.272$ ] than Hindu children and more likely to develop children from the central region [AOR: $1.510,95 \% \mathrm{Cl}(1.410,1.617)]$, east region [AOR: $1.077,95 \% \mathrm{Cl}(1.002,1.157)]$, and west region [AOR: $1.201,95 \% \mathrm{Cl}(1.095,1.317)]$. In both crude and adjusted analyses, the educational level of mothers/caregivers had no statistically significant association with childhood diarrhea.

In the crude analysis, the incidence of diarrhoeal morbidity had significantly 0.785 and 0.712 times less likely to develop among the children of mothers aged $25-34$ years [COR: $0.785,95 \% \mathrm{Cl}(0.759,0.811)$ ], 3549 years [COR: $0.712,95 \% \mathrm{Cl}(0.671,0.755)]$ respectively compared to younger aged mothers $(15-24$ years). Children in the rich household wealth index were significantly lower likely to have the diarrheal disease compared to those children in poor [COR: $0.942,95 \% \mathrm{Cl}(0.904,0.982)$ ] wealth index. The occurrences of diarrhea were significantly less likely to develop among children of mothers with normal [COR: $0.934,95 \% \mathrm{Cl}(0.901,0.968)$ ] and overweight \& obesity [COR: $0.860,95 \% \mathrm{Cl}(0.812,0.912)$ ] levels of Body Mass Index.

The occurrence of diarrhea was less likely to develop among children in 12-23 months [AOR: $0.928,95 \%$ $\mathrm{Cl}(0.876,0.983)], 24-35$ months [AOR: $0.579,95 \% \mathrm{Cl}(0.54,0.617)], 36-47$ months [AOR: $0.394,95 \% \mathrm{Cl}$ $(0.367,0.424)]$, and $49-59$ months [AOR: $0.313,95 \% \mathrm{Cl}(0.289,0.339)]$ compared to those children aged 0-11 months. The diarrhoeal disease was significantly 0.897 times less likely to prevail among female children [AOR: $0.897,95 \% \mathrm{Cl}(0.859,0.937)$ ]. The odds of emergent diarrhea was 1.135 [AOR: 1.135, 95\% $\mathrm{Cl}(1.074,1.201)]$ times higher among children with low birth weight compared to normal birth weight children. The odds of developing diarrhea was 1.097 [AOR: 1.097, 95\% $\mathrm{Cl}(1.047,1.149)$ ] times higher among undernourished children compared to well-nourished children. A high likelihood of diarrhea was found among those children whose mother had higher birth order (4+) [COR: 1.086, 95\% $\mathrm{Cl}(1.038,1.136)$ ] compared to first birth order children and more likely to found among those children [COR: $1.409,95 \% \mathrm{Cl}$ $(1.360,1.461)]$ who were currently breastfeeding (Table 5). 
Table 5

Multivariable binary logistic regression for analysis of socio-demographic and environmental factors associated with diarrhea among children aged 0-59 months in Rural India, NFHS-4, 2015-16

\begin{tabular}{|c|c|c|c|c|}
\hline Variables & $\begin{array}{l}\text { Crude } \\
\text { OR }\end{array}$ & $95 \% \mathrm{Cl}$ & $\begin{array}{l}\text { Adjusted } \\
\text { OR }\end{array}$ & $95 \% \mathrm{Cl}$ \\
\hline Social groups $(\mathrm{SC}+)$ & 1.00 & & 1.00 & \\
\hline ST & 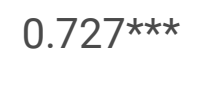 & $\begin{array}{l}(0.691 \text { - } \\
0.764)\end{array}$ & $0.811^{\star \star \star}$ & $\begin{array}{l}(0.755- \\
0.872)\end{array}$ \\
\hline OBC & 0.989 & $\begin{array}{l}(0.948- \\
1.031)\end{array}$ & $0.902^{\star \star \star}$ & $\begin{array}{l}(0.851- \\
0.956)\end{array}$ \\
\hline Other & 0.997 & $\begin{array}{l}(0.947- \\
1.050)\end{array}$ & 0.992 & $\begin{array}{l}(0.922- \\
1.067)\end{array}$ \\
\hline Religion (Hindu†) & 1.00 & & 1.00 & \\
\hline Muslim & $1.122^{\star \star \star}$ & $\begin{array}{l}(1.074- \\
1.173)\end{array}$ & 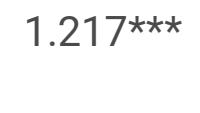 & $\begin{array}{l}(1.128- \\
1.313)\end{array}$ \\
\hline Other & $0.694 * * \star$ & $\begin{array}{l}(0.657- \\
0.732)\end{array}$ & $1.163^{\star \star \star}$ & $\begin{array}{l}(1.062- \\
1.272)\end{array}$ \\
\hline Current age of mother in years $(15-24 t)$ & 1.00 & & - & \\
\hline $25-34$ & $0.785^{\star \star \star}$ & $\begin{array}{l}(0.759- \\
0.811)\end{array}$ & - & - \\
\hline $35-49$ & $0.712^{\star \star \star}$ & $\begin{array}{l}(0.671- \\
0.755\end{array}$ & - & - \\
\hline $\begin{array}{l}\text { Education of mother/caregivers (Illiterate \& } \\
\text { primaryt) }\end{array}$ & 1.00 & & 1.00 & \\
\hline Secondary and above & 1.005 & $\begin{array}{l}(0.974- \\
1.037)\end{array}$ & 1.025 & $\begin{array}{l}(0.975- \\
1.077)\end{array}$ \\
\hline Wealth index (Poort) & 1.00 & & - & \\
\hline
\end{tabular}

OR= Odds Ratio, SC=Scheduled Caste, $S T=S c h e d u l e d$ Tribe, OBC= Other Backward Class, $h . h .=$ Households, $\mathrm{Cl}=$ Confidence Interval in parentheses,

*** $p<0.01, * \star p<0.05, * p<0.1$

$\dagger=$ Reference category. 


\begin{tabular}{|c|c|c|c|c|}
\hline Variables & $\begin{array}{l}\text { Crude } \\
\text { OR }\end{array}$ & $95 \% \mathrm{Cl}$ & $\begin{array}{l}\text { Adjusted } \\
\text { OR }\end{array}$ & $95 \% \mathrm{Cl}$ \\
\hline Middle & 0.984 & $\begin{array}{l}(0.946- \\
1.024)\end{array}$ & - & - \\
\hline Rich & $0.942^{\star \star \star}$ & $\begin{array}{l}(0.904- \\
0.982)\end{array}$ & - & - \\
\hline Maternal BMI (Underweight + ) & 1.00 & & - & \\
\hline Normal & $0.934^{\star \star \star}$ & $\begin{array}{l}(0.901 \text { - } \\
0.968)\end{array}$ & - & \\
\hline Overweight \& obesity & $0.860 * \star \star$ & $\begin{array}{l}(0.812- \\
0.912)\end{array}$ & - & - \\
\hline Region (Northt) & 1.00 & & 1.00 & \\
\hline Central & $1.454^{\star \star \star}$ & $\begin{array}{l}(1.390 \text { - } \\
1.520)\end{array}$ & 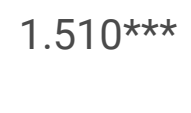 & $\begin{array}{l}(1.410- \\
1.617)\end{array}$ \\
\hline East & 0.987 & $\begin{array}{l}(0.939- \\
1.037)\end{array}$ & $1.077^{\star \star}$ & $\begin{array}{l}(1.002- \\
1.157)\end{array}$ \\
\hline North-east & $0.601 * * *$ & $\begin{array}{l}(0.564 \text { - } \\
0.640)\end{array}$ & $0.719 * \star \star$ & $\begin{array}{l}(0.647- \\
0.800)\end{array}$ \\
\hline West & 1.041 & $\begin{array}{l}(0.968 \text { - } \\
1.120)\end{array}$ & $1.201 * \star \star$ & $\begin{array}{l}(1.095- \\
1.317)\end{array}$ \\
\hline South & $0.696 * \star \star$ & $\begin{array}{l}(0.646- \\
0.750)\end{array}$ & $0.788 * \star \star$ & $\begin{array}{l}(0.718- \\
0.864)\end{array}$ \\
\hline Sex of child (Malet) & 1 & & 1.00 & \\
\hline Female & $0.914^{\star \star \star}$ & $\begin{array}{l}(0.886- \\
0.943)\end{array}$ & $0.897 \star \star \star$ & $\begin{array}{l}(0.859- \\
0.937)\end{array}$ \\
\hline Current age of child in months $(0-11+)$ & 1.00 & & 1.00 & \\
\hline
\end{tabular}

OR= Odds Ratio, SC=Scheduled Caste, $S T=S c h e d u l e d$ Tribe, OBC= Other Backward Class, $h . h .=$ Households, $\mathrm{Cl}=$ Confidence Interval in parentheses,

*** $p<0.01, * \star p<0.05, * p<0.1$

$\dagger=$ Reference category. 


\begin{tabular}{|c|c|c|c|c|}
\hline Variables & $\begin{array}{l}\text { Crude } \\
\text { OR }\end{array}$ & $95 \% \mathrm{Cl}$ & $\begin{array}{l}\text { Adjusted } \\
\text { OR }\end{array}$ & $95 \% \mathrm{Cl}$ \\
\hline $12-23$ & 0.968 & $\begin{array}{l}(0.928- \\
1.009)\end{array}$ & $0.928 * *$ & $\begin{array}{l}(0.876 \\
0.983)\end{array}$ \\
\hline $24-35$ & $0.602 * \star \star$ & $\begin{array}{l}(0.575- \\
0.631)\end{array}$ & $0.579 * \star \star$ & $\begin{array}{l}(0.543 \\
0.617)\end{array}$ \\
\hline $36-47$ & $0.393^{\star \star \star}$ & $\begin{array}{l}(0.373- \\
0.414)\end{array}$ & $0.394^{\star \star \star}$ & $\begin{array}{l}(0.367 \\
0.424)\end{array}$ \\
\hline $48-59$ & $0.318 * \star \star$ & $\begin{array}{l}(0.300- \\
0.336)\end{array}$ & $0.313^{\star \star \star}$ & $\begin{array}{l}(0.289 \\
0.339)\end{array}$ \\
\hline Birth order number $(1 \dagger)$ & 1.00 & & 1.00 & \\
\hline $2-3$ & $1.032^{\star}$ & $\begin{array}{l}(0.997- \\
1.069)\end{array}$ & 1.013 & $\begin{array}{l}(0.966 \\
1.063)\end{array}$ \\
\hline $3+$ & $1.086^{\star \star \star}$ & $\begin{array}{l}(1.038- \\
1.136)\end{array}$ & 1.039 & $\begin{array}{l}(0.967 \\
1.115)\end{array}$ \\
\hline Birth weight (Normalt) & 1.00 & & 1.00 & \\
\hline Low birth weight & $1.231^{\star \star \star}$ & $\begin{array}{l}(1.176- \\
1.289)\end{array}$ & $1.135^{\star \star \star}$ & $\begin{array}{l}(1.074 \\
1.201)\end{array}$ \\
\hline Nutritional status (Normal†) & 1.00 & & 1.00 & \\
\hline Undernourished & 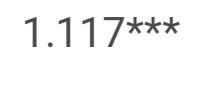 & $\begin{array}{l}(1.081- \\
1.155)\end{array}$ & 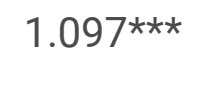 & $\begin{array}{l}(1.047 \\
1.149)\end{array}$ \\
\hline Currently breastfeeding (No†) & 1.00 & & - & \\
\hline Yes & $1.409 * \star \star$ & $\begin{array}{l}(1.360- \\
1.461)\end{array}$ & - & - \\
\hline Sources of drinking water (Unimprovedt) & 1.00 & & 1.00 & \\
\hline
\end{tabular}

OR= Odds Ratio, $S C=$ Scheduled Caste, $S T=S c h e d u l e d$ Tribe, $O B C=$ Other Backward Class, $h . h .=$ Households, $\mathrm{Cl}=$ Confidence Interval in parentheses,

*** $p<0.01, * \star p<0.05, * p<0.1$

$\dagger=$ Reference category. 


\begin{tabular}{|c|c|c|c|c|}
\hline Variables & $\begin{array}{l}\text { Crude } \\
\text { OR }\end{array}$ & $95 \% \mathrm{Cl}$ & $\begin{array}{l}\text { Adjusted } \\
\mathrm{OR}\end{array}$ & $95 \% \mathrm{Cl}$ \\
\hline Improved & $1.266^{\star \star \star}$ & $\begin{array}{l}(1.203- \\
1.332)\end{array}$ & $1.115^{\star \star \star}$ & $\begin{array}{l}(1.038 \text { - } \\
1.197)\end{array}$ \\
\hline Toilet facilities (Improved + ) & 1.00 & & 1.00 & \\
\hline Unimproved & $1.135^{\star \star \star}$ & $\begin{array}{l}(1.097- \\
1.174)\end{array}$ & 1.017 & $\begin{array}{l}(0.962- \\
1.075)\end{array}$ \\
\hline $\begin{array}{l}\text { Toilet facility shared with other h. h. (Not } \\
\text { sharedt) }\end{array}$ & 1.00 & & - & \\
\hline Shared & $1.288 * \star \star$ & $\begin{array}{l}(1.210- \\
1.371)\end{array}$ & - & - \\
\hline Disposal of youngest child's stools (Propert) & 1.00 & & 1.00 & \\
\hline Improper & 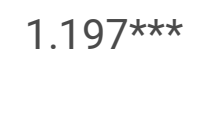 & $\begin{array}{l}(1.154- \\
1.242)\end{array}$ & $1.061^{\star \star}$ & $\begin{array}{l}(1.002- \\
1.124)\end{array}$ \\
\hline Main roof material (Concrete roof $\mathrm{t}$ ) & 1.00 & & 1.00 & \\
\hline Rudimentary roof & $1.140 * \star *$ & $\begin{array}{l}(1.092- \\
1.189)\end{array}$ & $1.113^{\star \star *}$ & $\begin{array}{l}(1.048 \text { - } \\
1.182)\end{array}$ \\
\hline Main floor material (Cleant) & 1.00 & & 1.00 & \\
\hline Unclean & $1.139 * \star \star$ & $\begin{array}{l}(1.102- \\
1.177)\end{array}$ & 1.026 & $\begin{array}{l}(0.974- \\
1.081)\end{array}$ \\
\hline Constant & & & $0.124 * \star *$ & $\begin{array}{l}(0.110- \\
0.141)\end{array}$ \\
\hline Pseudo R2 & & & 0.0367 & \\
\hline Log-likelihood & & & -29756.176 & \\
\hline Probability $x 2$ & & & 0.0000 & \\
\hline \multicolumn{5}{|c|}{$\begin{array}{l}\text { OR= Odds Ratio, } S C=\text { Scheduled Caste, } S T=S c h e d u l e d \text { Tribe, OBC= Other Backward Class, } h . h .= \\
\text { Households, Cl= Confidence Interval in parentheses, }\end{array}$} \\
\hline \multicolumn{5}{|l|}{$\star \star \star p<0.01, * \star p<0.05, * p<0.1$} \\
\hline$\dagger=$ Reference ca & & & & \\
\hline
\end{tabular}




\subsection{Household environmental predictors associated with diarrhea among under-five children}

A crude analysis of this study also found that the odds of developing diarrhea was 1.135 [COR: 1.135,9 $5 \% \mathrm{Cl}(1.097,1.174)]$ times higher among children whose families used unimproved toilet facilities compared to children whose families used improved toilet facilities. The odds of developing diarrhea was 1.288 [COR: $1.288,95 \% \mathrm{Cl}(1.210,1.371)]$ times higher among children of families who had shared toilet facilities with other households compared to children of families who hadn't shared toilet facilities with others. Children having houses of unclean floor materials were 1.139 [COR: 1.139, 95\% Cl $(1.102,1.177)$ ] times more likely to have suffered diarrhoeal disease than those children from houses with clean floor materials (Table 5).

Surprisingly, the emergent of diarrhea was 1.115 [AOR: 1.115, 95\% Cl $(1.038,1.197)$ ] times more likely to higher among children whose families used improved sources of drinking water compared to children of families who used unimproved water sources, the odds of increasing diarrhea was 1.061 [AOR: 1.061, $95 \% \mathrm{Cl}(1.002,1.124)]$ times more likely to diarrhea among children whose family improperly dispose of child's stools compared to those children whose family properly dispose of children's stools. Children having houses of rudimentary roof materials were 1.113 [AOR: 1.113, 95\% $\mathrm{Cl}(1.048,1.182)]$ times more likely to have suffered diarrhoeal disease than those children from houses with concrete roof materials (Table 5).

\section{Discussion}

The present study represents the investigation of different socio-demographic and environmental factors that were associated with diarrhea among the children of the below 5 years age group. Different factors were still determined for increasing the prevalence of this disease in the rural part of India. In India, the prevalence of diarrhea has been reduced in recent times but the hindrance of this disease is predominantly high in rural areas of India. According to NFHS-4 near about $9 \%$ of children whose age below 5 were adversely affected by diarrhea-related diseases.

This study depicts that social groups/caste are significantly associated with diarrhea disease. The children who belonged to the category of $\mathrm{ST}, \mathrm{OBC}$, and others were less likely to affect by this disease compared to SC children. A similar finding was also reported in other studies conducted in India [32]. The study also shows that religion also determining factor. The children who belonged to the religion of Muslim had a high risk of diarrhea disease than Hindu children. The result was also incorporated with the study of India [27, 32]. The higher risk of diarrhea disease among the scheduled caste and Muslim children might be the deprivation of safe drinking water and sanitation facilities [32]. The maternal factors such as maternal age and mother education were also analyzed by multivariate statistics but the relationship was not significant. On the other hand, crude analysis of these variables shows significant 
results, the women who had secondary or higher education level were less possibility of this disease among children as compared to the illiterate or primary level of mother's education. Similar findings are also consistent with the study of Ethiopia [25], India [33], and Bangladesh [20]. The crude analysis of the wealth index indicates that the rich and middle families had a lower risk of diarrhea disease than poor families, who had lower access to safe drinking water and sanitation facilities. Similar types of results were also found in previous studies of India $[27,33]$. The study also investigate that the underweight mothers had the high-risk factor of diarrhea among their children compared to normal-weight mothers. The underweight mother gave birth to malnourished and low immune system children who were susceptible to diseases like diarrhea and so on. The geographical location of the resident is considered to be an important factor for determining the diseases because the geographical location also determined the unequal availability of safe drinking water and sanitation. The study also indicates that the children from the central region had a higher risk of diarrhea disease than southern and western regions. In the same way, the children who belonged to the northeast region were less likely to develop this disease. The previous study of India also displayed the same type of result [34]. The study also reveals that the female children had a lower risk of diarrhea than the male children. This result is also incorporated with the result of the study of Bangladesh [20] and also in India [35] both the study represents a higher risk of diarrhoea among male children than female children. The risk of diarrhoea was very higher among the children of 12-23 months as compared to the higher current age. The risk of diarrheoa disease was very low among the higher age group children (36-59 months). The result is also incorporated with the study of India [36]. The lower age group or infant children were most affected by this disease because they had a weaker immune system.

The study also displayed that the women who had the higher birth order their children were more likely to have diarrhea disease than the lower birth order. The current study also focused on the birth weight factors which were revealed that the lower birth weight children had a higher possibility of diarrhea than the normal birth weight. The result is consistent with the previous study of India [37]. The undernourished and currently breastfeeding children were more likely affected by diarrhea disease. The finding is similar to the previous study of West Bengal [38].

The present study also displayed that the different environmental factors such as sources of drinking water, toilet facilities, child stool disposal, roof material, and floor material were associated with the diarrhea disease among the children of the under-five age group. A previous study of Ethiopia also represents a similar finding [16] The household with improved drinking water sources had a lower likelihood of diarrhea disease than unimproved drinking water sources. The result is also incorporated with the study of India [39]. Similarly improved toilet facilities reduced the higher risk of diarrhea disease. The study also stated that shared toilet facilities increased the higher risk of diarrhea among the children and the odds of improper child stool disposal also clearly revealed the higher likelihood of diarrhea among the children. The concrete roof materials of the house had a lower risk of diarrhea than the children who lived under the rudimentary roof. Another important fact also found that the children who were lived in dirty or unclean floor conditions had a higher likelihood of diarrhea disease. The reason 
behind this result might be the children attached to the dirty floor and for this reason, the pathogens transmitted to children. The result is consistent with the study of Ethiopia [25].

\section{Conclusion}

Diarrhea is still a very concerning health-related issue in the rural part of India. Although diarrhea-related mortality is decreasing the burden of this disease is present till now in the rural part of India. Different socio-demographic factors such as caste, religion, economic condition family, and women's education are significantly associated with the diarrhea disease. Scheduled Caste, Muslim, and economically backward family's children are more suffered by this disease. It is also noted that various environmental factors such as sources of drinking water, toilet facilities, stool disposal of the child, roof materials, and main floor materials of household are also significantly related to the risk factor of this disease. The bad sources of drinking water, dirty floor conditions, and unimproved toilet facilities are the vulnerable factors for diarrhea disease. The different socio-demographic and environmental factors should be improved or tackled by effective community education. The government and different NGOs should focus on improved drinking water sources, sanitation facility which may reduce the vulnerability of the disease. The existing different public health programs should focus to create awareness among the people about the importance of sanitation and cleanliness to mitigate diarrheal diseases.

\section{Abbreviations}

NFHS

National Family and Health Survey

AOR

Adjusted Odds Ratio

COR

Crude Odds Ratio

$\mathrm{Cl}$

Confidence Interval

NGO

Non-Government Organization

WHO

World Health Organization

GAPPD

Prevention and Control of Pneumonia and Diarrhoea

UNICEF

United Nations Children's Fun

ORS

Oral Rehydration solution

ICDS 
Integrated Child Development Services

ASHA

Accredited Social Health Activists

PPS

Probability Proportional to Size

\section{Declarations}

\section{Ethics approval and consent to participate}

The present used secondary data which is available in the public domain. The dataset has no identifiable information of the survey participants. Therefore, no ethical approval is required for conducting this study.

\section{Consent for publication}

Not applicable

\section{Availability of data and materials}

The general datasets are available from the Demographic Health Surveys (DHS) repository. Specifically, the data used for this study are available from the corresponding author on reasonable request.

\section{Competing interests}

The author declares that they have no competing interests.

\section{Funding}

The author did not receive any financial assistance from external funds.

\section{Authors' contributions}

\section{Credit authorship contributions}

The Author, JS, and PC conceptualization and designed the study, JS and SM both did data acquisition, performed the statistical data analysis, interpreted the results, and prepared the manuscript. The author PC also read and approved the final manuscript

\section{Acknowledgments}

The authors are thankful to International Institute for Population Sciences (IIPS), Mumbai for accepting the request to access the data in this study

\section{References}


1. WHO. Diarrhoeal disease. World Health Organization. 2017. https://www.who.int/news-room/factsheets/detail/diarrhoeal-disease. Accessed 29 Oct 2021.

2. Farthing MJG, Kelly P. Infectious diarrhoea. Medicine (Baltimore). 2007;35:251-6.

3. NHP. ORS Day. National Health Portal of India. 2015. https://www.nhp.gov.in. Accessed 29 Oct 2021.

4. UNICEF. Diarrhoea - UNICEF DATA. UNICEF. 2019. https://data.unicef.org/topic/childhealth/diarrhoeal-disease/. Accessed 29 Oct 2021.

5. UNICEF, WHO. Levels \& trends in Child mortality: Report 2015, Estimates Developed by the UN Interagency Group for Child Mortality Estimation. 2015.

6. UNICEF. UNICEF Data: Monitoring the situation of children and women. UNICEF. 2019. https://data.unicef.org/topic/child-health/diarrhoeal-disease/. Accessed 22 Nov 2021.

7. IIPS, ICF. National Family Health Survey - 4: 2015-16. International Institute for Population Sciences (IIPS), Mumbai, India. 2017. http://rchiips.org/nfhs/.

8. Mohanan M, Vera-Hernández M, Das V, Giardili S, Goldhaber-Fiebert JD, Rabin TL, et al. The know-do gap in quality of health care for childhood diarrhea and pneumonia in rural india. JAMA Pediatr. 2015;169:349-57.

9. WHO, UNICEF. Ending preventable child deaths from pneumonia and diarrhoea by 2025: The integrated Global Action Plan for Pneumonia and Diarrhoea (GAPPD). Geneva: WHO \& UNICEF. 2013.

10. Million Death Study Collaborators. Causes of neonatal and child mortality in India: a nationally representative mortality survey. Lancet. 2010;376:1853-60. doi:https://doi.org/10.1016/S01406736(10)61461-4.

11. Wardlaw T, Salama P, Brocklehurst C, Chopra M, Mason E. Diarrhoea: why children are still dying and what can be done. Lancet. 2010;375:870-2.

12. UNICEF, WHO. Diarrhoea: why children are still dying and what can be done. Geneva: UNICEF/WHO. 2009. https://www.who.int/publications/i/item/9789241598415. Accessed 29 Oct 2021.

13. Ministry of Health and Family Welfare. Health Ministry launches Intensified Diarrhoea Control Fortnight (IDCF)-Intensified efforts to reduce child deaths due to diarrhoea. Government of India. 2017. https://pib.gov.in/newsite/printrelease.aspx?relid=165631. Accessed 29 Oct 2021.

14. Shirole T. Indian Government Launches a Program to Reduce Diarrhea Deaths in the Country. Medindia, Indian Health News. 2015. https://www.medindia.net/news/indian-government-launchesa-program-to-reduce-diarrhea-deaths-in-the-country-151448-1.htm. Accessed 29 Oct 2021.

15. Kapil U. Integrated Child Development Services (ICDS) scheme: A program for holistic development of children in India. Indian J Pediatr. 2002;69:597-601.

16. Mihrete TS, Alemie GA, Teferra AS. Determinants of childhood diarrhea among underfive children in Benishangul Gumuz regional state, north West Ethiopia. BMC Pediatr. 2014;14:1-9. doi:https://doi.org/10.1186/1471-2431-14-102.

17. Mohammed S, Tamiru D. The Burden of Diarrheal Diseases among Children under Five Years of Age in Arba Minch District, Southern Ethiopia, and Associated Risk Factors: A Cross-Sectional Study. Int 
Sch Res Not. 2014;2014:1-6.

18. Lakshminarayanan S, Jayalakshmy R. Diarrheal diseases among children in India: Current scenario and future perspectives. J Nat Sci Biol Med. 2015;6:24-8.

19. Azage M, Kumie A, Worku A, Bagtzoglou AC. Childhood diarrhea in high and low hotspot districts of Amhara Region, northwest Ethiopia: a multilevel modeling. J Health Popul Nutr. 2016;35:1-14.

20. Sarker AR, Sultana M, Mahumud RA, Sheikh N, Van Der Meer R, Morton A. Prevalence and Health Care-Seeking Behavior for Childhood Diarrheal Disease in Bangladesh. Glob Pediatr Heal. 2016;3:2333794X1668090.

21. Anteneh ZA, Andargie K, Tarekegn M. Prevalence and determinants of acute diarrhea among children younger than five years old in Jabithennan District, Northwest Ethiopia, 2014. BMC Public Health. 2017;17:1-8. doi:10.1186/s12889-017-4021-5.

22. Asfaha KF, Tesfamichael FA, Fisseha GK, Misgina KH, Weldu MG, Welehaweria NB, et al. Determinants of childhood diarrhea in Medebay Zana District, Northwest Tigray, Ethiopia: A community based unmatched case-control study. BMC Pediatr. 2018;18:1-9.

23. Getachew A, Guadu T, Tadie A, Gizaw Z, Gebrehiwot M, Cherkos DH, et al. Diarrhea Prevalence and Sociodemographic Factors among Under-Five Children in Rural Areas of North Gondar Zone, Northwest Ethiopia. Int J Pediatr. 2018;2018:1-8.

24. Zedie F, Kassa D. Socio-economic, behavioral and environmental factors associated with diarrhea among under five children in health development and non-health development army member mothers in Wondogenet, south Ethiopia. Heal Educ Care. 2018;3:1-8.

25. Melese B, Paulos W, Astawesegn FH, Gelgelu TB. Prevalence of diarrheal diseases and associated factors among under-five children in Dale District, Sidama zone, Southern Ethiopia: A cross-sectional study. BMC Public Health. 2019;19:1-10.

26. Workie GY, Akalu TY, Baraki AG. Environmental factors affecting childhood diarrheal disease among under-five children in Jamma district, South Wello zone, Northeast Ethiopia. BMC Infect Dis. 2019;19:804.

27. Vijayan B, Ramanathan M. Prevalence and clustering of diarrhoea within households in India: Some evidence from NFHS-4, 2015-16. J Biosoc Sci. 2020;:1-13.

28. Abuzerr S, Nasseri S, Yunesian M, Hadi M, Zinszer K, Mahvi AH, et al. Water, sanitation, and hygiene risk factors of acute diarrhea among children under five years in the Gaza Strip. J Water, Sanit Hyg Dev. 2020;10:111-23. doi:https://doi.org/10.2166/washdev.2019.072.

29. Godana W, Mengiste B. Environmental factors associated with acute diarrhea among children under five years of age in derashe district, Southern Ethiopia. Sci J Public Heal. 2013;1:119-24. doi:10.11648/j.sjph.20130103.12.

30. Adane M, Mengistie B, Kloos H, Medhin G, Mulat W. Pone.0182783.Pdf. 2017;:1-18.

31. Nsabimana J, Mureithi C, Habtu M. Factors contributing to diarrheal diseases among children less than five years in Nyarugenge District, Rwanda. Rwanda. 2017. doi:10.4172/2329-891X.1000238. 
32. Mallick R, Mandal S, Chouhan P. Impact of sanitation and clean drinking water on the prevalence of diarrhea among the under-five children in India. Child Youth Serv Rev. 2020;118 June:105478. doi:10.1016/j.childyouth.2020.105478.

33. Paul P. Socio-demographic and environmental factors associated with diarrhoeal disease among children under five in India. BMC Public Health. 2020;20.

34. Kamath A, Shetty K, Unnikrishnan B, Kaushik S, Rai SN. Prevalence, patterns, and predictors of diarrhea: a spatial-temporal comprehensive evaluation in India. Springer. 2018;18:1-10.

35. Avachat SS, Phalke VD, Phalke DB, Aarif SM, Kalakoti P. A cross-sectional study of sociodemographic determinants of recurrent diarrhoea among children under five of rural area of Western Maharashtra, India. Australas Med J. 2011;4:72.

36. Ahmed SF, Farheen A, Muzaffar A, Attoo GM. Prevalence of diarrhoeal disease, its seasonal and age variation in under-fives in Kashmir, India. Int J Health Sci (Qassim). 2008;2:126. https://www.ncbi.nlm.nih.gov/pmc/articles/pmc3068726/. Accessed 24 Oct 2021.

37. Stanly AM, Sathiyasekaran BW, Palani G. A population based study of acute diarrhoea among children under 5 years in a rural community in South India. sriramachandra.edu.in. 2009;1:17. https://www.sriramachandra.edu.in/university/pdf/research/journals/aug_2008.pdf\#page=9. Accessed 24 Oct 2021.

38. Gupta A, Sarker G, Rout AJ, Mondal T, Pal R. Risk correlates of diarrhea in children under 5 years of age in slums of Bankura, West Bengal. J Glob Infect Dis. 2015;7:23. doi:10.4103/0974-777X.150887.

39. Lakshminarayanan S, Jayalakshmy R. Diarrheal diseases among children in India: Current scenario and future perspectives. J Nat Sci Biol Med. 2015;6:24. doi:10.4103/0976-9668.149073. 